\title{
9 ROLE OF MAGNETIC RESONANCE IMAGING TO DIFFERENTIATE BETWEEN GLIOBLASTOMA AND PRIMARY CENTRAL NERVOUS SYSTEM LYMPHOMA:
}

RETROSPECTIVE STUDY

Mahmoud Lofty El Sheikh, Doaa Mokhtar mohamed Emara, Tarek Ahmed Rayan,* Nahla Ibrahim Nasr Mohamed

Department of Radiodiagnosis, Department of Neurosurgery,* Faculty of Medicine, Alexandria University.

\section{Introduction}

Preoperative differentiation of primary central nervous system lymphomas (PCNSLs) and glioblastomas is of high clinical relevance because treatment strategies for glioblastoma and PCNSL vary substantially. In patients with glioblastoma, gross total resection followed by radiation therapy and chemotherapy with temozolomide is the treatment of choice, whereas patients with PCNSL usually undergo stereotactic biopsy followed by high dose methotrexate-based chemotherapy.

Standard magnetic resonance (MR) imaging sequences allow differentiation for most patients because PCNSL in immunocompetent patients usually appears as a homogeneous enhancement on contrast material-enhanced T1-weighted MR images, but glioblastomas usually exhibit a ring or ringlike zone of contrast enhancement around a hypointense central area of necrosis. A T2 hyperintense edema surrounds both tumors. Diffusion weighted imaging a well established advanced an MR technique has been reported to improve the differential diagnosis between PCNSL and GBM. Water diffusion can be measured with a parameter of between PCNSL and GBM. Water diffusion can be measured with a parameter of
apparent diffusion coefficient (ADC). Highly cellular tumors like PCNSL show areas of restricted diffusion with low ADC values, thus ADC is regarded as a marker of tumor cellularity.

\section{Aim of the work}

The aim of the study was to assess the role of magnetic resonance imaging in differentiation between glioblastoma and primary central nervous system lymphoma.

\section{Sulbjects and Methods}

\section{PATIENTS:}

This retrospective study was carried on 20 patients with histologically proven glioblastoma and primary central nervous system lymphoma presented to the Radiology Department, Main university Hospital, Alexandria university.

\section{METHODS:}

All patients included in the study underwent the following:

Complete history taking.

Reviewing Magnetic resonance imaging.

T1, T2 (axial, sagittal and coronal) and diffusion weighted images

- T1 axial post- contrast images.

Reviewing histopathological assessment

-statistical analysis of the data.

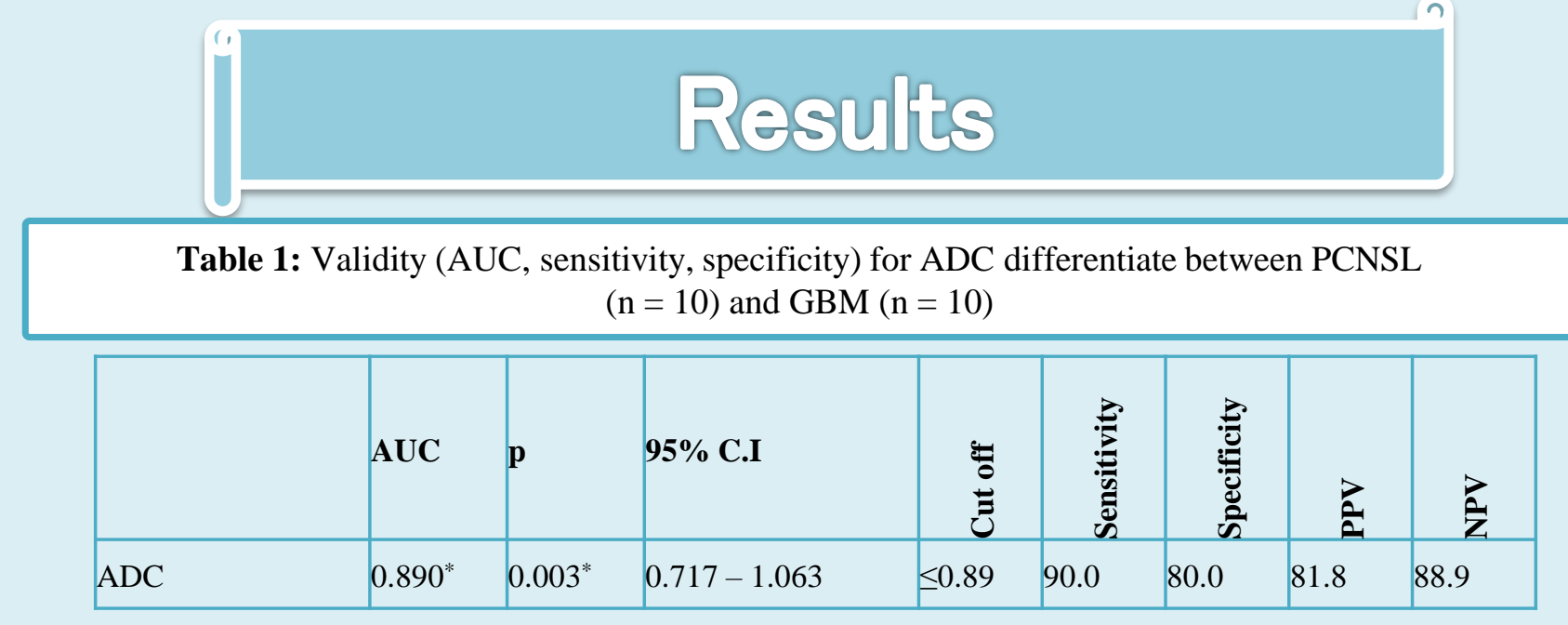

$\begin{array}{lll}\text { AUC: Area Under a Curve } & \text { p value: Probability value } & \text { CI: Confidence Intervals } \\ \text { NPV: Negative predictive value } & \text { PPV: Positive predictive value } & * \text { *: Statistically significant at } \mathrm{p} \leq 0.05\end{array}$

Table 2: MRI findings in PCNSL and GBM at initial evaluation

\begin{tabular}{|c|c|c|c|}
\hline Mri finding & & PCNSL & GBM \\
\hline Site & $\begin{array}{l}\text { Supratentorial } \\
\text { Infratentorial } \\
\text { Supra- and infra tentorial }\end{array}$ & $\begin{array}{l}90 \% \\
10 \% \\
0 \%\end{array}$ & $\begin{array}{l}100 \% \\
0 \% \\
0 \%\end{array}$ \\
\hline Multiplicity of lesions & $\begin{array}{l}\text { Solitary } \\
\text { Multiple }\end{array}$ & $\begin{array}{l}60 \% \\
40 \%\end{array}$ & $\begin{array}{l}100 \% \\
0 \%\end{array}$ \\
\hline Pattern of enhancement & $\begin{array}{l}\text { Homogenous } \\
\text { Non homogenous } \\
\text { Marginal }\end{array}$ & $\begin{array}{l}50 \% \\
50 \% \\
0 \%\end{array}$ & $\begin{array}{l}0 \% \\
50 \% \\
50 \%\end{array}$ \\
\hline $\begin{array}{l}\text { CC infiltration } \\
\text { BG involvement }\end{array}$ & & $\begin{array}{l}20 \% \\
10 \%\end{array}$ & $\begin{array}{l}20 \% \\
10 \%\end{array}$ \\
\hline DWI & $\begin{array}{l}\text { Free diffusion } \\
\text { Restricted in solid parts }\end{array}$ & $\begin{array}{l}0 \% \\
100 \%\end{array}$ & $\begin{array}{l}20 \% \\
80 \%\end{array}$ \\
\hline Mean ADC value & & $0.7568 \pm 0.10$ & $1.0095 \pm 0.17$ \\
\hline $\begin{array}{l}\begin{array}{l}\text { Presence of vasogenic } \\
\text { edema }\end{array} \\
\end{array}$ & & $90 \%$ & $90 \%$ \\
\hline Intratumoral bleeding & & $0 \%$ & $40 \%$ \\
\hline
\end{tabular}
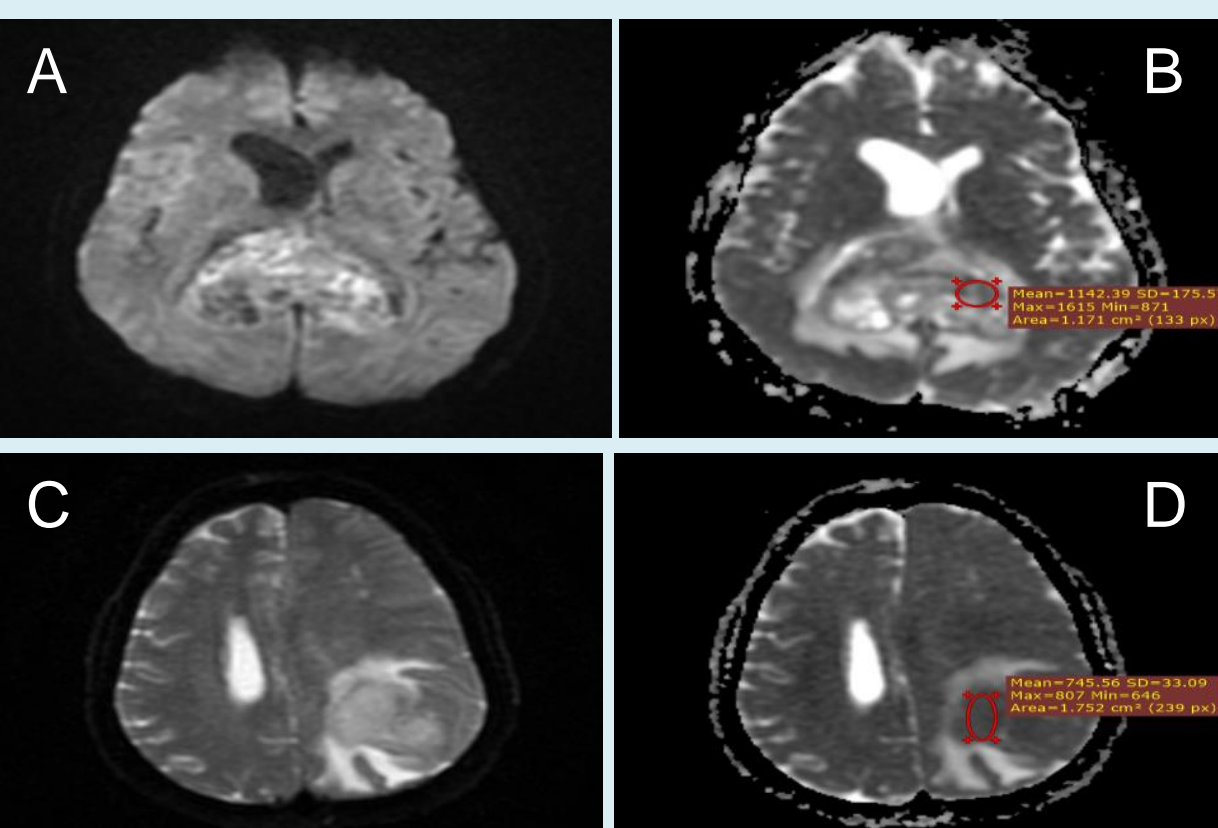

C

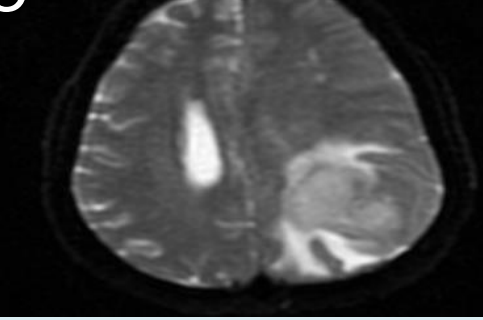

Figure: (a\&b) GBM.DWI showing diffusion restriction with mean ADC value of $1.142 \times 10-3$ $\mathrm{mm} 2 / \mathrm{sec}(\mathrm{sed}$
$\mathrm{mm} 2 / \mathrm{sec}$.

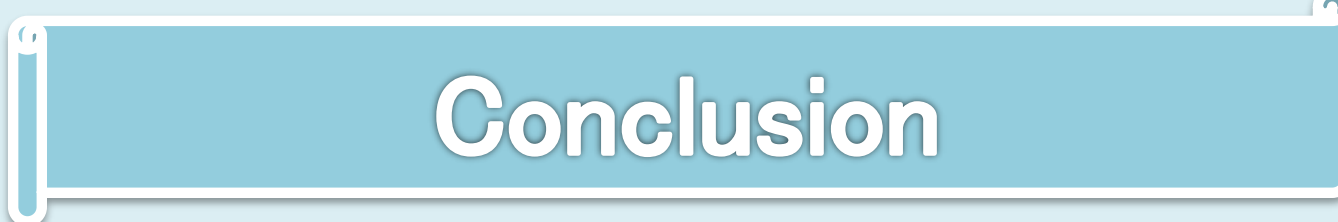

-conventional MRI is able to differentiate between GBM and PCNSL in some cases where GBM appears as a heterogenous lesion with marginal heterogenous enhancement and central necrosis while PCNSL appears as homogenously enhancing lesion with no necrosis

DWI/ADC is an accurate parameter in differentiation between both tumors due to relative minimum ADC value in PCNSL and A cut-off value of $0.89 \times 10-3 \mathrm{~mm} 2 / \mathrm{sec}$ has been suggested with $90 \%$ sensitivity and $80 \%$ specificity.

DWI is an easy technique to perform and fast to postprocess should be incorporated in the MR protocol of all intracranial tumors.

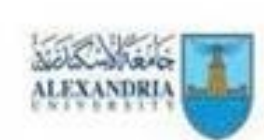$$
\text { 2021@Alexandria Faculty of Medicine }
$$

MEDICINE $\mid$ FacuTY OP 